\title{
On a Competitive System of Rational Difference Equations
}

\author{
Mehmet Gümüş ${ }^{1}$ \\ ${ }^{1}$ Zonguldak Bülent Ecevit University, Faculty of Science and Arts, Department of Mathematics, 67100, Zonguldak, Turkey
}

\author{
Article Info \\ Keywords: Equilibrium point, Global \\ stability, Rate of convergence, System \\ of difference equations \\ 2010 AMS: 39A10, 40A05 \\ Received: 20 November 2019 \\ Accepted: 18 December 2019 \\ Available online: 26 December 2019
}

\begin{abstract}
This paper aims to investigate the global stability and the rate of convergence of positive solutions that converge to the equilibrium point of the system of difference equations in the modeling competitive populations in the form
\end{abstract}

$$
x_{n+1}^{(1)}=\frac{\alpha x_{n-2}^{(1)}}{\beta+\gamma \prod_{i=0}^{2} x_{n-i}^{(2)}}, x_{n+1}^{(2)}=\frac{\alpha_{1} x_{n-2}^{(2)}}{\beta_{1}+\gamma\left(\prod_{i=0}^{2} x_{n-i}^{(1)}\right.}, n=0,1, \ldots
$$

where the parameters $\alpha, \beta, \gamma, \alpha_{1}, \beta_{1}, \gamma_{1}$ are positive numbers and the initial conditions $x_{-i}^{(1)}, x_{-i}^{(2)}$ are arbitrary non-negative numbers for $i \in\{0,1,2\}$.

\section{Introduction}

Difference equation or discrete dynamical system is a diverse field which impacts almost every branch of pure and applied mathematics. Every difference equation determines a dynamical system and vice versa. Recently, there has been a big interest in studying difference equation systems. One of the reasons for this is a necessity for some techniques which can be used in investigating equations arising in mathematical models describing real life situations in population biology, economics, probability theory, genetics, psychology, see $[10,11]$. Therefore, the asymptotic behavior of solutions of the system for rational difference equations has received huge interest, see $[1,2,3,4,5,6,7,8,9,13]$.

This paper deals with the following two-dimensional system

$$
x_{n+1}^{(1)}=\frac{\alpha x_{n-2}^{(1)}}{\beta+\gamma \prod_{i=0}^{2} x_{n-i}^{(2)}}, x_{n+1}^{(2)}=\frac{\alpha_{1} x_{n-2}^{(2)}}{\beta_{1}+\gamma\left(\prod_{i=0}^{2} x_{n-i}^{(1)}\right.}, n=0,1, \ldots
$$

where the parameters $\alpha, \beta, \gamma, \alpha_{1}, \beta_{1}, \gamma_{1}$ are positive numbers and the initial conditions $x_{-i}^{(1)}, x_{-i}^{(2)}$ are arbitrary non-negative numbers for $i \in\{0,1,2\}$. Actually, in [15] some dynamical behaviors of the system (1.1) has been studied. But, we notice that the authors have not examined various properties of system (1.1), namely, the global stability, the rate of convergence and the asymptotic behavior. Our aim in this paper is to give a complete picture as regards the global behavior of positive solutions of system (1.1). That is, we here study the global asymptotic stability of zero equilibrium and the rate of convergence of solutions.

The following the boundedness and the local stability results have obtained in [15].

Lemma 1.1. $\left(\bar{x}_{1}, \bar{x}_{2}\right)=(0,0)$ is always an equilibrium point of system (1.1).

Theorem 1.2. If both $\frac{\alpha}{\beta}<1$ and $\frac{\alpha_{1}}{\beta_{1}}<1$, then every positive solution of system (1.1) is bounded.

Theorem 1.3. If both $\frac{\alpha}{\beta}<1$ and $\frac{\alpha_{1}}{\beta_{1}}<1$, then the zero equilibrium point of system (1.1) is locally asymptotically stable.

In the present paper, we will provide some results about the global behavior and the rate of convergence of positive solutions that converge to the zero equilibrium point of the system (1.1), in the regions of parameters described in Theorem 1.3. In addition to this, we will present the 
use of Poincaré's Theorem and a devolopment of Perron's Theorem to conclude the precise asymptotics of positive solutions that converge to the equilibrium.

Consider the following one-dimensional system of difference equations

$$
\left.\begin{array}{l}
x_{n+1}=f_{1}\left(x_{n}, y_{n}\right), n=0,1, \ldots \\
y_{n+1}=f_{2}\left(x_{n}, y_{n}\right), n=0,1, \ldots
\end{array}\right\}
$$

where $f_{1}, f_{2}$ are continuous functions that maps some set $I$ into $I$. The set $I$ is an interval of real numbers. System (1.2) is competitive if $f_{1}(x, y)$ is non-decreasing in $x$ and non-increasing in $y$ and $f_{2}(x, y)$ is non-increasing in $x$ and non-decreasing in $y$. System (1.2) is called anti-competitive system, if the functions $f_{1}$ and $f_{2}$ have monotonic character opposite to the monotonic character in competitive system. It is well know that the dynamical properties of competitive populations has received great interest from both theoretical and mathematical biologists [14] due to its universal commonness. Competitive and anti-competitive systems were studied by many authors (see [1, 4, 5, 8]). Especially, studying the rate of convergence of solutions of some systems of difference equations is a topic of big interest [2, 3, 9].

We state that the following theorems give precise information about the asymptotics of linear non-autonomous difference equations. Consider the scalar $m t h$-order linear difference equation

$$
y_{n+m}+p_{1}(n) y_{n+m-1}+\ldots+p_{m}(n) y_{n}=0
$$

where $m$ is a positive integer and $p_{i}: \mathbb{Z}^{+} \rightarrow \mathbb{C}$ for $i \in\{1, \ldots, m\}$. Suppose that

$$
q_{i}=\lim _{n \rightarrow \infty} p_{i}(n), \text { for } i=1,2, \ldots, m,
$$

exist in $\mathbb{C}$. For the following limitting equation of (1.3)

$$
y_{n+m}+q_{1} y_{n+m-1}+\ldots+q_{m} y_{n}=0,
$$

the asymptotics of solutions of (1.3) are given the following results. See [10, 13].

Theorem 1.4. (Poincaré's Theorem) Consider (1.3) based on the condition (1.4). Let $\lambda_{i}$ for $i=1, \ldots, m$ be the roots of the characteristic equation

$$
\lambda^{m}+q_{1} \lambda^{m-1}+\ldots+q_{m}=0
$$

of the limiting equation (1.5) under the condition that $\left|\lambda_{i}\right| \neq\left|\lambda_{j}\right|$ for $i \neq j$. If $x_{n}$ is a positive solution of (1.3), then either $x_{n}=0$ for all large $n$ or there exists an index $j \in\{1, \ldots, m\}$ such that

$$
\lim _{n \rightarrow \infty} \frac{x_{n+1}}{x_{n}}=\lambda_{j} .
$$

The releated results were obtained by Perron, and one of Perron's results was improved by Pituk, see [13].

Theorem 1.5. Assume that (1.4) holds. If $x_{n}$ is a positive solution of (1.3), then either eventually $x_{n}=0$ or

$$
\lim _{n \rightarrow \infty} \sup \left(\left|x_{n j}\right|\right)^{1 / n}=\left|\lambda_{j}\right| \text {, }
$$

where $\lambda_{1}, \ldots, \lambda_{m}$ are the roots (not necessarily distinct) of the characteristic equation (1.6).

Consider

$$
Y_{n+1}=[A+B(n)] Y_{n}
$$

where $Y_{n}$ is an $m$-dimensional vector, $A \in C^{m \times m}$ is a constant matrix and

$$
B: \mathbb{Z}^{+} \rightarrow C^{m \times m}
$$

is a matrix function satisfying

$$
\|B(n)\| \rightarrow 0, \text { when } n \rightarrow \infty,
$$

where $\|$.$\| denotes any matrix norm which is associated with the vector norm \|$.$\| . See [12].$

Theorem 1.6. (Pituk) Suppose that condition (1.8) holds for system (1.7). If $Y_{n}$ is a solution of (1.7), then either

$$
Y_{n}=0
$$

for all large $n$ or

$$
\theta=\lim _{n \rightarrow \infty}\left\|Y_{n}\right\|^{1 / n}
$$

exists and $\theta$ is equal to the modulus one of the eigenvalues of the matrix $A$.

Theorem 1.7. (Pituk) Suppose that condition (1.8) holds for system (1.7). If $Y_{n}$ is a solution of (1.7), then either

$$
Y_{n}=0
$$

for all large $n$ or

$$
\theta=\lim _{n \rightarrow \infty} \frac{\left\|Y_{n+1}\right\|}{\left\|Y_{n}\right\|}
$$

exists and $\theta$ is equal to the modulus one of the eigenvalues of the matrix $A$. 


\section{Global Behavior of the system (1.1)}

In this section, we investigate the global behavior of the system (1.1).

Theorem 2.1. If both $\frac{\alpha}{\beta}<1$ and $\frac{\alpha_{1}}{\beta_{1}}<1$, then the zero equilibrium point of system (1.1) is globally asymptotically stable.

Proof. We know by Theorem 1.3 that the zero equilibrium point $\left(\bar{x}_{1}, \bar{x}_{2}\right)=(0,0)$ of the system (1.1) is locally asymptotically stable. So, it suffices to prove for any solution $\left\{\left(x_{n}^{(1)}, x_{n}^{(2)}\right)\right\}_{n=-2}^{\infty}$ of system (1.1) that

$$
\lim _{n \rightarrow \infty}\left(x_{n}^{(1)}, x_{n}^{(2)}\right)=(0,0) .
$$

From the boundedness result system (1.1) it is clear that it is sufficient to prove that $\left\{\left(x_{n}^{(1)}, x_{n}^{(2)}\right)\right\}_{n=-2}^{\infty}$ is decreasing. We have that

$$
0 \leq x_{n+1}^{(1)}=\frac{\alpha x_{n-2}^{(1)}}{\beta+\gamma \prod_{i=0}^{2} x_{n-i}^{(2)}}<\frac{\alpha}{\beta} x_{n-2}^{(1)}
$$

and

$$
0 \leq x_{n+1}^{(2)}=\frac{\alpha_{1} x_{n-2}^{(2)}}{\beta_{1}+\gamma_{1} \prod_{i=0}^{2} x_{n-i}^{(1)}}<\frac{\alpha_{1}}{\beta_{1}} x_{n-2}^{(2)} .
$$

By induction on $n$, one has

$$
0 \leq x_{3 n+i}^{(1)}<\left(\frac{\alpha}{\beta}\right)^{n+1} x_{-3+i}^{(1)}, i=1,2,3
$$

and

$$
0 \leq x_{3 n+i}^{(2)}<\left(\frac{\alpha}{\beta}\right)^{n+1} x_{-3+i}^{(2)}, i=1,2,3 .
$$

Thus, for $\frac{\alpha}{\beta}<1$ and $\frac{\alpha_{1}}{\beta_{1}}<1$, we can have

$$
\lim _{n \rightarrow \infty}\left(x_{n}^{(1)}, x_{n}^{(2)}\right)=(0,0) .
$$

This completes the proof.

\section{Rate of Convergence}

In this section, we will characterize the rate of convergence of a solution that converges to the equilibrium point

$$
M=\left(\bar{x}_{1}, \bar{x}_{2}\right)=(0,0)
$$

of the system (1.1).

Using Theorem 1.6 and 1.7, we obtain the following rate of convergence result.

Theorem 3.1. Suppose that $\frac{\alpha}{\beta}<1$ and $\frac{\alpha_{1}}{\beta_{1}}<1$. Let $\left\{\left(x_{n}^{(1)}, x_{n}^{(2)}\right)\right\}_{n=-2}^{\infty}$ be any positive solution of the system (1.1) such that

$$
\begin{aligned}
& \lim _{n \rightarrow \infty} x_{n}^{(1)}=\bar{x}_{1}, \\
& \lim _{n \rightarrow \infty} x_{n}^{(2)}=\bar{x}_{2}
\end{aligned}
$$

where $M=\left(\bar{x}_{1}, \bar{x}_{2}\right)$ and $M$ is globally asymptotically stable. Then, the error vector

$$
E_{n}=\left(\begin{array}{c}
e_{n}^{1} \\
e_{n-1}^{1} \\
e_{n-2}^{1} \\
e_{n}^{2} \\
e_{n-1}^{2} \\
e_{n-2}^{2}
\end{array}\right)_{6 \times 1}=\left(\begin{array}{c}
x_{n}^{(1)}-\bar{x}_{1} \\
x_{n-1}^{(1)}-\bar{x}_{1} \\
x_{n-2}^{(1)}-\bar{x}_{1} \\
x_{n}^{(2)}-\bar{x}_{2} \\
x_{n-1}^{(2)}-\bar{x}_{2} \\
x_{n-2}^{(2)}-\bar{x}_{2}
\end{array}\right)_{6 \times 1}
$$

of every positive solution of the system (1.1) satisfies both of the following asymptotic relations:

$$
\begin{aligned}
& \lim _{n \rightarrow \infty}\left\|E_{n}\right\|^{1 / n}=\left|\lambda_{i} J_{F}(M)\right|, \text { for some } i=1,2, \ldots, 6 \\
& \lim _{n \rightarrow \infty} \frac{\left\|E_{n+1}\right\|}{\left\|E_{n}\right\|}=\left|\lambda_{i} J_{F}(M)\right|, \text { for some } i=1,2, \ldots, 6
\end{aligned}
$$

where

$$
\left|\lambda_{i} J_{F}(M)\right|
$$

is equal to the modulus one of the eigenvalues of the Jacobian matrix evaluated at the equilibrium point M. 
Proof. Let $\left\{\left(x_{n}^{(1)}, x_{n}^{(2)}\right)\right\}_{n=-2}^{\infty}$ be any positive solution of the system (1.1) such that

$$
\lim _{n \rightarrow \infty} x_{n}^{(1)}=\bar{x}_{1}
$$

and

$$
\lim _{n \rightarrow \infty} x_{n}^{(2)}=\bar{x}_{2}
$$

To find the error terms, we have

$$
\begin{aligned}
& x_{n+1}^{(1)}-\bar{x}_{1}=\sum_{i=0}^{2} A_{i}\left(x_{n-i}^{(1)}-\bar{x}_{1}\right)+\sum_{i=0}^{2} B_{i}\left(x_{n-i}^{(2)}-\bar{x}_{2}\right) \\
& x_{n+1}^{(2)}-\bar{x}_{2}=\sum_{i=0}^{2} C_{i}\left(x_{n-i}^{(1)}-\bar{x}_{1}\right)+\sum_{i=0}^{2} D_{i}\left(x_{n-i}^{(2)}-\bar{x}_{2}\right) .
\end{aligned}
$$

Set

$$
\begin{aligned}
& e_{n}^{1}=x_{n}^{(1)}-\bar{x}_{1}, \\
& e_{n}^{2}=x_{n}^{(2)}-\bar{x}_{2} ;
\end{aligned}
$$

it follows that

$$
\begin{aligned}
& e_{n+1}^{1}=\sum_{i=0}^{2} A_{i} e_{n-i}^{1}+\sum_{i=0}^{2} B_{i} e_{n-i}^{2} \\
& e_{n+1}^{2}=\sum_{i=0}^{2} C_{i} e_{n-i}^{1}+\sum_{i=0}^{2} D_{i} e_{n-i}^{2}
\end{aligned}
$$

where

$$
\begin{aligned}
& A_{0}=0, A_{1}=0, A_{2}=\frac{\alpha}{\beta+\gamma \prod_{i=0}^{2} x_{n-i}^{(2)}}, \\
& B_{0}=-\frac{\alpha \gamma x_{n-2}^{(1)} x_{n-1}^{(2)} x_{n-2}^{(2)}}{\left(\beta+\gamma \prod_{i=0}^{2} x_{n-i}^{(2)}\right)^{2}}=-\frac{\alpha \gamma x_{n-2}^{(1)} x_{n}^{(2)} x_{n-2}^{(2)}}{\left(\beta+\gamma \prod_{i=0}^{2} x_{n-i}^{(2)}\right)^{2}}, B_{2}=-\frac{\alpha \gamma x_{n-2}^{(1)} x_{n}^{(2)} x_{n-1}^{(2)}}{\left(\beta+\gamma \prod_{i=0}^{2} x_{n-i}^{(2)}\right)^{2}} \\
& C_{0}=-\frac{\alpha_{1} \gamma_{1} x_{n-2}^{(2)} x_{n-1}^{(1)} x_{n-2}^{(1)}}{\left(\beta_{1}+\gamma_{1} \prod_{i=0}^{2} x_{n-i}^{(1)}\right)^{2}} C_{1}=-\frac{\alpha_{1} \gamma_{1} x_{n-2}^{(2)} x_{n}^{(1)} x_{n-2}^{(1)}}{\left(\beta_{1}+\gamma_{1} \prod_{i=0}^{2} x_{n-i}^{(1)}\right)^{2}}, C_{2}=-\frac{\alpha_{1} \gamma_{1} x_{n-2}^{(2)} x_{n}^{(1)} x_{n-1}^{(1)}}{\left(\beta_{1}+\gamma_{1} \prod_{i=0}^{2} x_{n-i}^{(1)}\right)^{2}} \\
& D_{0}=0, D_{1}=0, D_{2}=\frac{\alpha_{1}}{\beta_{1}+\gamma_{1} \prod_{i=0}^{2} x_{n-i}^{(1)}} .
\end{aligned}
$$

Taking the limits, it is clear that

$$
\begin{aligned}
& \lim _{n \rightarrow \infty} A_{0}=0, \lim _{n \rightarrow \infty} A_{1}=0 \text { and } \lim _{n \rightarrow \infty} A_{2}=\frac{\alpha}{\beta+\gamma \bar{x}_{2}^{3}} \\
& \lim _{n \rightarrow \infty} B_{0}=-\frac{\alpha \gamma \bar{x}_{1} \bar{x}_{2}^{2}}{\left(\beta+\gamma \bar{x}_{2}^{3}\right)^{2}}, \lim _{n \rightarrow \infty} B_{1}=-\frac{\alpha \gamma \bar{x}_{1} \bar{x}_{2}^{2}}{\left(\beta+\gamma \bar{x}_{2}^{3}\right)^{2}} \text { and } \lim _{n \rightarrow \infty} B_{2}=-\frac{\alpha \gamma \bar{x}_{1} \bar{x}_{2}^{2}}{\left(\beta+\gamma \bar{x}_{2}^{3}\right)^{2}}, \\
& \lim _{n \rightarrow \infty} C_{0}=-\frac{\alpha_{1} \gamma_{1} \bar{x}_{1}^{2} \bar{x}_{2}}{\left(\beta_{1}+\gamma_{1} \bar{x}_{1}^{3}\right)^{2}}, \lim _{n \rightarrow \infty} C_{1}=-\frac{\alpha_{1} \gamma_{1} \bar{x}_{1}^{2} \bar{x}_{2}}{\left(\beta_{1}+\gamma_{1} \bar{x}_{1}^{3}\right)^{2}} \text { and } \lim _{n \rightarrow \infty} C_{2}=-\frac{\alpha_{1} \gamma_{1} \bar{x}_{1}^{2} \bar{x}_{2}}{\left(\beta_{1}+\gamma_{1} \bar{x}_{1}^{3}\right)^{2}}, \\
& \lim _{n \rightarrow \infty} D_{0}=0, \lim _{n \rightarrow \infty} D_{1}=0 \text { and } \lim _{n \rightarrow \infty} D_{2}=\frac{\alpha_{1}}{\beta_{1}+\gamma_{1} \bar{x}_{1}^{3}} .
\end{aligned}
$$

That is

$$
\begin{aligned}
& A_{2}=\frac{\alpha}{\beta+\gamma \bar{x}_{2}^{3}}+\varsigma_{n}, \quad B_{0}=-\frac{\alpha \gamma \bar{x}_{1} \bar{x}_{2}^{2}}{\left(\beta+\gamma \bar{x}_{2}^{3}\right)^{2}}+\tau_{n}, \quad B_{1}=-\frac{\alpha \gamma \bar{x}_{1} \bar{x}_{2}^{2}}{\left(\beta+\gamma \bar{\gamma}_{2}^{3}\right)^{2}}+v_{n}, \quad B_{2}=-\frac{\alpha \gamma \bar{x}_{1} \bar{x}_{2}^{2}}{\left(\beta+\gamma \bar{x}_{2}^{3}\right)^{2}}+\delta_{n} \\
& C_{0}=-\frac{\alpha_{1} \gamma_{1} \bar{x}_{1}^{2} \bar{x}_{2}}{\left(\beta_{1}+\gamma_{1} \bar{x}_{1}^{3}\right)^{2}}+\lambda_{n}, \quad C_{1}=-\frac{\alpha_{1} \gamma_{1} \bar{x}_{1}^{2} \bar{x}_{2}}{\left(\beta_{1}+\gamma_{1} \bar{x}_{1}^{3}\right)^{2}}+\varepsilon_{n}, \quad C_{2}=-\frac{\alpha_{1} \gamma_{1} \bar{x}_{1}^{2} \bar{x}_{2}}{\left(\beta_{1}+\gamma_{1} \bar{x}_{1}^{3}\right)^{2}}+\eta_{n}, \quad D_{2}=\frac{\alpha_{1}}{\beta_{1}+\gamma_{1} \bar{x}_{1}^{3}}+\varphi_{n}
\end{aligned}
$$

where $\varsigma_{n} \rightarrow 0, \tau_{n} \rightarrow 0, v_{n} \rightarrow 0, \delta_{n} \rightarrow 0, \lambda_{n} \rightarrow 0, \varepsilon_{n} \rightarrow 0, \eta_{n} \rightarrow 0, \varphi_{n} \rightarrow 0$ for $n \rightarrow \infty$.

Thus, the limitting system of error terms about the equilibrium $M$ can be written as follows:

$$
E_{n+1}=(C+D(n)) E_{n},
$$

where $E_{n}=\left(e_{n}^{1}, e_{n-1}^{1}, e_{n-2}^{1}, e_{n}^{2}, e_{n-1}^{2}, e_{n-2}^{2}\right)^{T}$,

$$
C=\left(\begin{array}{cccccc}
0 & 0 & \frac{\alpha}{\beta} & 0 & 0 & 0 \\
1 & 0 & 0 & 0 & 0 & 0 \\
0 & 1 & 0 & 0 & 0 & 0 \\
0 & 0 & 0 & 0 & 0 & \frac{\alpha_{1}}{\beta_{1}} \\
0 & 0 & 0 & 1 & 0 & 0 \\
0 & 0 & 0 & 0 & 1 & 0
\end{array}\right)_{6 \times 6}, D_{n}=\left(\begin{array}{cccccc}
0 & 0 & \varsigma_{n} & \tau_{n} & v_{n} & \delta_{n} \\
0 & 0 & 0 & 0 & 0 & 0 \\
0 & 0 & 0 & 0 & 0 & 0 \\
\lambda_{n} & \varepsilon_{n} & \eta_{n} & 0 & 0 & \varphi_{n} \\
0 & 0 & 0 & 0 & 0 & 0 \\
0 & 0 & 0 & 0 & 0 & 0
\end{array}\right)_{6 \times 6}
$$

and $\|D(n)\| \rightarrow 0$, when $n \rightarrow \infty$. This completes the proof. 


\section{Conflict of Interests}

The author declares that there is no conflict of interests regarding the publication of this manuscript.

\section{Acknowledgement}

The author would like to thank the Editor and the anonymous referees for their careful reading and constructive suggestions.

\section{References}

[1] D. Burgic, M. R. S. Kulenovic and M. Nurkanovic, Global Dynamics of a Rational System of Difference Equations in the plane, Comm. Appl. Nonlinear Anal., 15 (2008), 71-84

[2] D. Burgić and M. Nurkanović, The Rational System of Nonlinear Difference Equations in the Modeling Competitive Populations, 15th International Research/Expert Conference, Trends in the Devolpment of Machinery and Associated Tehnology, TMT, (2011).

[3] D. Burgić and A. Huskanovic, The Rational System of Equations in the Modeling Anti-Competitive Populations, 18th International Research/Expert Conference, Trends in the Devolpment of Machinery and Associated Tehnology, TMT, (2014).

[4] A. Brett, M. Garic-Demirovic, M. R. S. Kulenovic and M. Nurkanovic, Global behavior of two competitive rational systems of difference equations in the plane, Commun. Appl. Nonlinear Anal., 16 (2009), 1-18.

[5] D. Clark, M. R. S. Kulenovic, and J. F. Selgrade, Global asymptotic behavior of a two dimensional difference equation modelling competition, Nonlinear Analysis. Theory, Methods \& Applications, 52 (7) (2003), 1765-1776.

[6] M. Gocen and M. Guneysu, The Global Attractivity of some ratinal difference equations, J. Comp. Anal. Appl., 25(7) (2018), 1233-1243.

[7] M. Gocen and A. Cebeci, On the periodic solutions of some systems of higher order difference equations, Rocky Mountain Journal of Mathematics, 48(3) (2018), 845-858.

[8] T. F. Ibrahim, Two-dimensional fractional system of nonlinear difference equations in the modeling competitive populations, International Journal of Basic \& Applied Sciences, 12 (5) (2012), 103-121.

[9] S. Kalabušić and M. R. S. Kulenović, Rate of convergence of solutions of rational difference equation of second order, Advances in Difference Equations, 2 (2004): 1-19

[10] V. Kocić and G. Ladas, Global behavior of nonlinear difference equations of higher order with applications, Kluwer Academic Publishers, Dordrecht, (1993)

[11] M. R. S. Kulenović and G. Ladas, Dynamics of second order rational difference equations, Chapman \& Hall/CRC, Boca Raton, London (2001).

[12] M. R. S. Kulenović and O. Merino, Discrete Dynamical Systems and Difference Equations with Mathematica, Chapman \& Hall/CRC, Boca Raton, London (2002)

[13] M. Pituk, More on Poincaré's and Perron's Theorems for Difference Equations, Journal of Difference Equations and Applications, 8 (3) (2002), 201-216.

[14] J. F. Selgrade and M. Ziehe, Convergence to equilibrium in a genetic model with differential viability between the sexes, Journal of Mathematical Biology, 25 (5) (1987), 477-490.

[15] Q. Zhang, L. Yang and J. Liu, Dynamics of a system of rational third-order difference equation, Advances in Difference Equations, 136 (2012), 1-6. 